Abstract P-059 Table 2 Adjusted outcomes

\begin{tabular}{|c|c|c|c|c|c|c|c|c|c|}
\hline \multirow[b]{2}{*}{ Factor } & \multicolumn{3}{|c|}{ Poor Outcome } & \multicolumn{3}{|c|}{ Length of Stay } & \multicolumn{3}{|c|}{ Any Complication } \\
\hline & $\mathrm{OR}$ & $95 \% \mathrm{Cl}$ & $\mathrm{P}$ & $\exp ($ Beta, B) & $95 \% \mathrm{Cl}$ & $\mathrm{P}$ & $\mathrm{OR}$ & $95 \% \mathrm{Cl}$ & $P$ \\
\hline Race & 1.32 & $1.07,1.62$ & 0.008 & 0.04 & $0.03,0.06$ & $<0.001$ & 0.94 & $0.80,1.10$ & 0.4 \\
\hline Black & 0.77 & $0.60,0.99$ & 0.043 & 0.04 & $0.02,0.05$ & $<0.001$ & 1.02 & $0.85,1.23$ & 0.8 \\
\hline Hispanic & 0.6 & $0.42,1.09$ & 0.12 & 0.02 & $0.01,0.05$ & 0.2 & 0.81 & $0.56,1.15$ & 0.2 \\
\hline Asian & REF & - & - & REF & - & - & REF & - & - \\
\hline \multicolumn{10}{|l|}{ White } \\
\hline Age & 1.04 & $1.04,1.05$ & $<0.001$ & -0.01 & $-0.05,0.04$ & 0.8 & 1.01 & $1.00,1.01$ & $<0.001$ \\
\hline Sex & 1.11 & $0.94,1.31$ & 0.2 & 0.01 & $0.00,0.02$ & 0.044 & 1.25 & $1.10,1.43$ & $<0.001$ \\
\hline Female & REF & - & - & REF & - & - & REF & - & - \\
\hline \multicolumn{10}{|l|}{ Male } \\
\hline Clip & 2.15 & $1.83,2.52$ & $<0.001$ & 0.27 & $0.26,0.28$ & $<0.001$ & 0.77 & $0.68,0.87$ & $<0.001$ \\
\hline Elective & 0.67 & $0.57,0.80$ & $<0.001$ & -0.30 & $-0.32,-0.29$ & $<0.001$ & 1.16 & $1.01,1.33$ & 0.032 \\
\hline Obese & 1.13 & $0.90,1.40$ & 0.3 & 0.00 & $-0.01,0.02$ & 0.8 & 0.95 & $0.80,1.13$ & 0.6 \\
\hline Diabetic & 1.34 & $1.10,1.64$ & 0.004 & 0.00 & $-0.02,0.01$ & 0.9 & 1.20 & $1.02,1.40$ & 0.025 \\
\hline Tobacco & 0.85 & $0.72,1.01$ & 0.071 & 0.00 & $-0.01,0.01$ & $>0.9$ & 0.96 & $0.85,1.09$ & 0.6 \\
\hline Severity of IIIness & 190 & 130,286 & $<0.001$ & 0.78 & $0.76,0.80$ & $<0.001$ & 68.6 & $54.5,86.6$ & $<0.001$ \\
\hline Extreme & 26.1 & $18.1,38.9$ & $<0.001$ & 0.46 & $0.45,0.48$ & $<0.001$ & 16.7 & $13.7,20.3$ & $<0.001$ \\
\hline Major & 4.96 & $3.43,7.41$ & $<0.001$ & 0.14 & $0.13,0.15$ & $<0.001$ & 3.97 & $3.33,4.76$ & $<0.001$ \\
\hline Moderate & REF & - & - & REF & - & - & REF & - & - \\
\hline \multicolumn{10}{|l|}{ Minor } \\
\hline Hospital Status & 0.64 & $0.22,2.16$ & 0.4 & -0.11 & $-0.19,-0.02$ & 0.015 & 2.04 & $0.71,6.94$ & 0.2 \\
\hline Urban Teaching & 0.75 & $0.25,2.60$ & 0.6 & -0.11 & $-0.20,-0.02$ & 0.013 & 2.23 & $0.77,7.68$ & 0.2 \\
\hline Urban Non-Teaching & REF & - & - & REF & - & - & REF & - & - \\
\hline Rural & & & & & & & & & \\
\hline
\end{tabular}

$<0.001)$ patients experienced slightly longer length of stay compared to White patients. The odds of experiencing any complication were similar across races.

Conclusion In this nationwide analysis, racial disparities were present in that Black patients were at increased odds of poor outcomes and both Black and Hispanic patients experience longer lengths of stay when compared to White patients after adjusting for several possible confounding factors. In the shared effort to provide equitable care, an important first step is analyzing and acknowledging the differences in objective outcomes as racial disparities in neurosurgery. Understanding the specific factors underlying the differences, such as possible differences in care access, is the next step to improve health equity in cerebrovascular neurosurgery.

Disclosures S. Koester: None. A. Yengo-Kahn: None. M. Feldman: None. M. Lan: None. P. Patel: None. A. Churchwell: None. R. Chitale: None.

\section{P-060 APPLICATION OF NON-DESTRUCTIVE MECHANICAL CHARACTERIZATION TESTING FOR CREATING IN VITRO VESSEL MODELS WITH MATERIAL PROPERTIES SIMILAR TO HUMAN NEUROVASCULATURE}

K Lewis*, J Vigil, N Norris, W Merritt, T Becker. Mechanical Engineering: Bioengineering Program Mechanical Engineering (ME), Northern Arizona University, Flagstaff, AZ

\subsection{6/neurintsurg-2021-SNIS.96}

Introduction/Purpose Vessel models are a first step in developing endovascular medical devices. These models are often madefrom glass or silicone, which do not accurately represent the mechanical properties of human vasculartissue, limiting their use to basic training and proof-of-concept testing. This study outlines methods toquantify the mechanical properties of both human vascular tissue and synthetic biomaterials for creatingrepresentative vessel models.
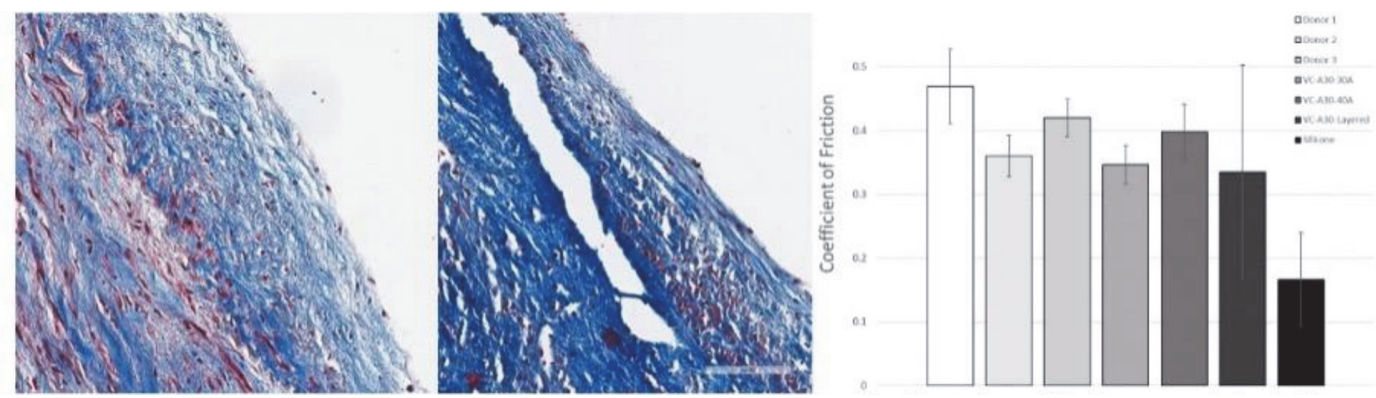

Abstract P-060 Figure 1 Left - non-destructive mechanical testing of human tissue. No disruption of tunica or endothelial layer. Middle destructive mechanical testing, apparent tunica and endothelial disruptions. Right - coefficient of friction lubricity of the human donor, compared to VC-A30 (dark gray) and silicone (black) 
Materials and Methods Human vascular tissue was assessed and compared to standard silicone and new UV-cured polymers (VC-A30). Vessel materials were characterized with eight mechanical tests: compressive, shear, and tensiledynamic elastic modulus, Poisson's ratio, hardness, radial compression, compliance, and lubricity. Half ofthese testing methods were nondestructive, allowing for multiple mechanical and histologicalcharacterizations of the same human tissue sample.

Results Histological evaluation of cellular and extracellular matrix of the human vessels showed the dynamicmoduli and Poison's ratio tests were non-destructive (figure $1 \mathrm{Left}$ ), whereas the destructive hardnesstest created significant tearing of the vessel layers (figure 1 Middle). Fluid absorption by VC-A30 showedstatistically significant softening of mechanical properties, stabilizing after 4 days in phosphate-bufferedsaline (PBS). VCA30 exhibited statistically similar results to human vasculature, with\% error less than $29 \%$, in 5 of 8 mechanical tests, versus 1 of 8 for standard silicone. Human vessel lubricity (determinesdevice trackablility within a vessel) statistically matched the lubricity of all the VC-A30 samples (figure 1 Right).

Conclusion VC-A30 provides a new option for creating translucent in vitro vascular models with anatomically-relevantproperties. VC-A30 can be formed into highly accurate models with specific mechanical properties usingthe latest 3D-printing techniques. These new vessel analogs may simulate patient-specific vessel diseasestates, improve surgical training models, accelerate the development of new endovascular devices, andultimately reduce dependencies on animal models.

Disclosures K. Lewis: None. J. Vigil: None. N. Norris: None. W. Merritt: None. T. Becker: 1; C; NIH grant \#5R42NS097069-03.

\section{Electronic poster abstracts}

\section{E-001 ENDOVASCULAR MANAGEMENT OF TRAUMATIC INTRACRANIAL ANEURYSMS FROM CLOSED HEAD INJURY}

${ }^{1} \mathrm{~A}$ White*, ${ }^{2} \mathrm{C}$ Roark, ${ }^{2} \mathrm{D}$ Case, ${ }^{2} \mathrm{Z}$ Folzenlogen, ${ }^{3} \mathrm{D}$ Kumpe, ${ }^{4} \mathrm{D}$ Ding, ${ }^{2} \mathrm{~J}$ Seinfeld. ${ }^{1}$ Radiology, UT Southwestern Medical Center, Dallas, TX; ${ }^{2}$ Neurosurgery, University of Colorado, Aurora, $\mathrm{CO} ;{ }^{3}$ Radiology, University of Colorado, Aurora, $\mathrm{CO}^{4}{ }^{4}$ Neurosurgery, University of Louisville, Louisville, KY

\subsection{6/neurintsurg-2021-SNIS.97}

Introduction/Purpose Traumatic intracranial aneurysms (TICAs) comprise a rare and particularly dangerous subset of cerebral aneurysms that can be difficult to both diagnose and manage, owing to their locations, morphologies, and presence of concomitant traumatic brain injury (TBI).

Materials and Methods We retrospectively reviewed internal databases comprised of intracranial aneurysms treated at two U.S. academic medical centers from 2010 to 2019. Patients with aneurysms of the intracranial circulation as a result of blunt force trauma treated with endovascular methods were included. All patients underwent initial non-contrast head CT, non-invasive vascular imaging, and diagnostic cerebral angiography. Clinical and radiographic data were recorded.

Results Between January 2010 and December 2019, a total of 8 patients with traumatic intracranial aneurysms treated with endovascular methods were included. Patients were aged 9-62 years (mean 35.5) and most were male $(n=5)$. Five of 8 patients (62\%) experienced acute intracranial hemorrhage due to aneurysm rupture. All patients but one were found to have an associated fracture on initial CT, including the ipsilateral petrous bone $(n=4)$, anterior clinoid process $(n=1)$, posterior clinoid $(n=2)$, sphenoid body $(n=6)$, clivus $(n=2)$, and carotid canal $(n=3)$, while 6 of 8 patients were noted to have sphenoid hemosinus on initial imaging. The most frequently involved vessel was the internal carotid artery $(I C A ; n=6)$, including 2 cavernous segments, 2 supraclinoid segments, 1 ophthalmic segment, and 1 communicating segment. The other vessels involved include the anterior cerebral artery (pericallosal; $n=1$ ) and the posterior inferior cerebellar artery (tonsillomedullary segment; $n=1)$. Aneurysm sizes ranged from 2-8 $\mathrm{mm}$ (mean, $4.4 \mathrm{~mm}$ ). Three of 8 aneurysms were treated with flow diversion (FD), one of which had adjuvant coil embolization, while 3 aneurysms were treated with balloon-assisted coiling (BAC). The 2 non-ICA aneurysms were treated with parent vessel sacrifice (PVS), one with liquid embolics and coil embolization, the other with coil embolization alone. Complete angiographic cure was achieved in 5 of 8 patients. Three aneurysm recurrences were found on follow-up imaging, one of which presented as re-rupture, and all of which were retreated. Re-treatment modalities included FD alone, FD with adjuvant coil embolization, and direct coil embolization alone. Two of 3 treated recurrences were completely cured on angiographic follow-up, while one expired before sufficient time to judge treatment efficacy had passed. Despite technical success in the overwhelming majority of cases, half of the patients were discharged with a poor functional outcome (mRS 3-6).

Conclusion TICAs may form acutely or in a delayed manner following blunt force trauma and occur most frequently on the ICA owing to its proximity to the rigid bony and dural structures of the skull base. The presence of cranial fractures and sphenoid hemosinus warrants prompt intracranial vascular imaging, particularly in a TBI patient with acute neurological decline or new neurologic deficit. Endovascular management is effective, particularly FD, which has emerged as an attractive alternative to PVS in carefully selected patients. Outcomes tend to be poor despite technically successful endovascular treatment, and further investigations are needed to show which patients might benefit the most.

Disclosures A. White: None. C. Roark: None. D. Case: 2; C; Medtronic. Z. Folzenlogen: None. D. Kumpe: None. D. Ding: None. J. Seinfeld: 2; C; Medtronic.

\section{E-002 IMPACT OF PROCEDURAL TECHNIQUES ON CLINICAL OUTCOMES IN TREATING LARGE VESSEL OCCLUSION WITH ENDOVASCULAR THERAPY IN THE ASSIST REGISTRY}

${ }^{1} \mathrm{R}$ Gupta*, ${ }^{2} \mathrm{~A}$ Rai, ${ }^{3} \mathrm{D}$ Liebeskind, ${ }^{4} \mathrm{~A}$ Krajina, ${ }^{5} \mathrm{M}$ Psychogios, ${ }^{6} \mathrm{~T}$ Krings, ${ }^{7} \mathrm{~W}$ Yoon, ${ }^{8} \mathrm{O}$ Zaidat, ${ }^{9} \mathrm{~A}$ Puri, ${ }^{10} \mathrm{~A}$ Sarraj, ${ }^{11} \mathrm{M}$ Möhlenbruch. ${ }^{1}$ WMG Neurosurgery, Wellstar Health System, Marietta, GA; ${ }^{2}$ Department of Neuroradiology, West Virginia University, Morgantown, WV; ${ }^{3}$ Department of Neurology, University of California Los Angeles, Los Angeles, $C A ;{ }^{4}$ Department of Radiology, University Hospital Hradec Kralove, Hradec Králové, CZECH Republic; ${ }^{5}$ Department of Radiology, University Hospital Basel, Basel, Switzerland; ${ }^{6}$ Department of Neuroradiology, University of Toronto, Toronto Western Hospital, Toronto, ON, Canada; 'Department of Neuroradiology, Chonnam National University Hospital, Gwangju, Korea, Republic of; ${ }^{8}$ Department of Neurology, Saint Vincent Mercy Medical Center, Toledo, $\mathrm{OH}^{9}{ }^{9}$ Department of Radiology, University of Massachusetts Medical Center, Worcester, MA; ${ }^{10}$ Department of Neurology, University of Texas Houston Medical Center, Houston, TX; ${ }^{11}$ Department of Neuroradiology, University of Heidelberg Medical Center, Heidelberg, Germany

10.1136/neurintsurg-2021-SNIS.98 